\title{
ARTICLE
}

\section{A solar flare simulation and dosimetry facility at a proton therapy cyclotron}

\author{
Bhaskar Mukherjee $^{\mathrm{a} * \S}$, Reinhard Hentschel ${ }^{\mathrm{a}}$, Carolina Llina ${ }^{\mathrm{a}}$, Xiaoning Ding ${ }^{\mathrm{a}}$, Sam Tripathy ${ }^{\mathrm{b}}$, Chandra Sunil $^{\mathrm{b}}$ \\ and Pradip Kumar Sarkar ${ }^{\mathrm{b}}$ \\ ${ }^{a}$ West German Proton Therapy Centre Essen (WPE) gGmbH, Hufelandstrasse 55, D-45147 Essen, Germany; ${ }^{b}$ Health Physics \\ Division, Bhabha Atomic Research Centre, Mumbai 400094, India
}

During extravehicular activities (EVA) astronauts are exposed to intense radiation from solar flare events (SFE). Hence, the radiation shielding efficacy of space suits, in particular the helmets and extra vehicular visor assembly (EVVA) are imperative to safety of astronauts and ultimate success of the space mission. At West German Proton Therapy Centre Essen (WPE) we have replicated the historical solar flare spectra by manipulating the treatment planning system (TPS) of the $230 \mathrm{MeV}$ proton-therapy cyclotron and developed novel proton irradiation and dosimetry methods for the testing of space borne devices as mentioned above. We have used a single foil of radiochromic film (type EBT 2) to estimate the proton depth dose distribution in the human eye. The optimal thickness of EVVA (acryl glass) for the protection from solar flares was estimated to be $2.35 \mathrm{~cm}$. A maximum SFE proton dose up to $20 \mathrm{~Gy}$ found to be well assessed using the EBT 2 films.

Keywords: extra vehicular activity; extra vehicular visor assembly; low earth orbit; international space station; medical cyclotron; proton therapy; radiochromic film; solar flare

\section{Introduction}

The solar flare events (SFE), composed of protons of a wide energy distribution are highly sporadic. During space mission astronauts often undertake extravehicular activities (EVA). For example during construction and maintenance of International Space Station (ISS), placed in the orbit at 51.6 degrees inclination the astronauts are exposed to intense, life-threatening radiation from SFE. Hence, the radiological shielding efficacy of space suit and extravehicular visor assembly (EVVA) become highly imperative to human space missions [1].

The quality testing of space suits and helmets of both US American and Russian origins using conventional X-ray CT scan and a $155 \mathrm{MeV}$ synchrotron produced proton beam at Loma Linda University has been described elsewhere [2]. A research group at Francis H. Burr Proton Therapy Center reported the construction and operation principle of a dedicated rotating range modulator wheel for solar flare simulation [3].

At WPE we have replicated the historical solar flare spectra [4, 5] (Figure 1) by manipulating the treatment planning system (TPS) [6] of $230 \mathrm{MeV}$ proton therapy cyclotron and developed a novel solar flare simulation facility and dosimetry technique using radiochromic films. We have planned to use the system for the testing of smaller space borne devices like helmets and EVVA.

\footnotetext{
*Corresponding author. Email: bhamukh@gmail.com
}

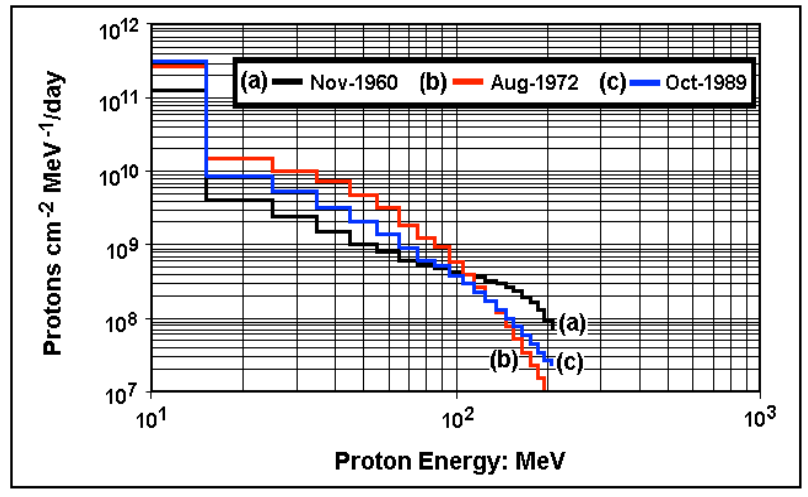

Figure 1. Historical solar flare event (SFE) spectra adopted from literature [4]. The spectra have been binned for the purpose of proton dose calculations.

\section{Materials and methods}

\subsection{Ocular proton dosimetry}

Prior to our own irradiation experiment at WPE cyclotron we have established the method proton dose calculation in the most radiosensitive part of the eye, i.e. the lens as highlighted in Figure 2 (left). We created a physical dosimetry model by replacing the lens and cornea-aqueous humor with $2 \mathrm{~mm}$ and $4 \mathrm{~mm}$ thick polystyrene plates respectively (right). The occupational

$\S$ Research Fellow, The University of Sydney, Australia 
dose limit for eye was considered to be $150 \mathrm{mSv} / \mathrm{year}$ [7].

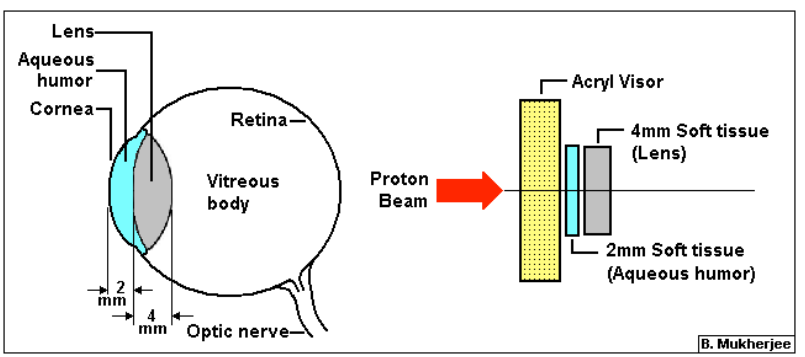

Figure 2. The anatomy of human eye showing its essential parts used in dosimetry calculations (left) and the physical dosimetry model (right). The position of acryl glass plate emulating the visor is also indicated.

The proton dose $\left(\mathrm{D}_{\mathrm{P}}\right)$ and average LET $\left(\mathrm{LET}_{\mathrm{av}}\right)$ in eye Lens (soft tissue equivalent) were calculated according to equations 1 and 2 respectively.

$$
\begin{gathered}
D_{p}=\frac{1.602 \times 10^{-13} a_{l} \sum^{i} \Delta E_{i} \phi_{i}}{m_{l}}(\mathrm{~Gy}) \\
L E T_{a v}=\frac{\sum^{i} \Delta E_{i} \phi_{i}}{t_{l} \sum^{i} \phi_{i}} \times 10^{-3}(\mathrm{keV} / \mathrm{m})
\end{gathered}
$$

Where, $a_{l}, m_{l}$ and $t_{l}$ represent the cross sectional area $\left(0.79 \mathrm{~cm}^{2}\right)$, mass $(314 \mathrm{mg})$ and thickness of the lens respectively [7]. $\Delta \mathrm{E}_{\mathrm{i}}$ and $\phi_{\mathrm{i}}$ stand for energy loss of protons of $i^{\text {th }}$ bin in the lens and corresponding proton fluence respectively.

\subsection{Proton irradiation at WPE cyclotron}

Utilizing the uniform scanning (US) treatment modality on IBA universal nozzle we have developed the proton irradiation set up as depicted in Figure 3.

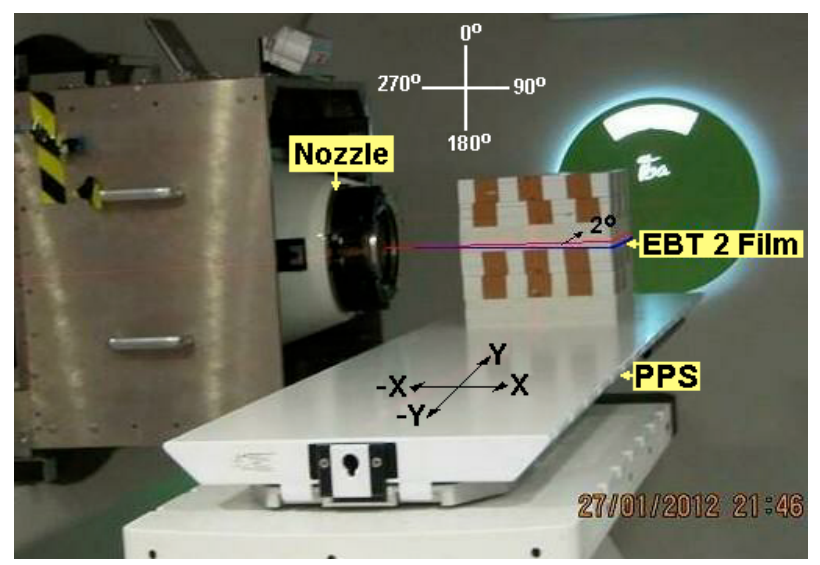

Figure 3. Showing the proton irradiation and dosimetry facility developed for the testing of (small) space suit components at the Proteus 230 Medical Cyclotron of WPE.
For the SFE simulation experiment we have used a polyethylene (water/tissue equivalent) plate phantom made of a stack of 30 plates $\left(25 \times 25 \mathrm{~cm}^{2}\right)$. The phantom was placed on the patient positioning system (PPS) and irradiated laterally, by rotating the beam delivery nozzle at $272^{\circ}$ with vertical plane. A $10 \times 10 \mathrm{~cm}^{2}$ proton field at uniform scanning (US) mode was implemented. The proton energy variation was carried out using the range shifter device of the energy selection system (ESS) located at the Proteus 230 Medical Cyclotron. The cyclotron was situated in an adequately shielded bunker at a distance of about $110 \mathrm{~m}$ from the irradiation set up (Figure 3). The entire irradiation procedure, including beam direction adjustment, phantom-positioning on the PPS (Figure 3) as well as proton energy variation were performed by programming the treatment planning system (TPS) similar to a typical cancer therapy protocol with protons [6].

The proton dose calibration was performed by using a single sheet $\left(19 \times 25 \mathrm{~cm}^{2}\right)$ of radiochromic film (Type: EBT 2, Manufacturer: International Specialty Products, NJ 07470, USA) was sandwiched between the two polystyrene plates at central plane of the phantom. Evidently the tissue equivalent EBT 2 film mimics a thin cross section through the eye (Figure 2) suitable for proton dose profile estimation. Prior to proton bombardment with a beam cross-section of $10 \times 10 \mathrm{~cm}^{2}$ the beam delivery nozzle was tilted to an angle of $272^{\circ}$ (Figure 3). This small angular deviation from the "ideal" horizontal plane (EBT 2 Film) prevents proton scattering in the boundary layers of air clogged between the polystyrene plates and EBT 2 film.

In order to emulate and study the shielding efficacy of the EVVA, a $6 \mathrm{~mm}$ thick acryl glass plate was used to block half of the proton beam profile. The proton doses were delivered in a 7 step histogram mode: $(x)=>15$ $\mathrm{MeV} / 100 \mathrm{~Gy}$, (a) =>58 MeV/20 Gy, (b) =>95 MeV/1.0 Gy, (c) =>128 MeV/0.1 Gy, (d) =>165 MeV/0.08 Gy, (e) $=>195 \mathrm{MeV} / 0.03 \mathrm{~Gy},\left(\mathrm{f}^{*}\right)=>225 \mathrm{MeV} / 0.015 \mathrm{~Gy}$ emulating a solar flare depicted in Figure 4.

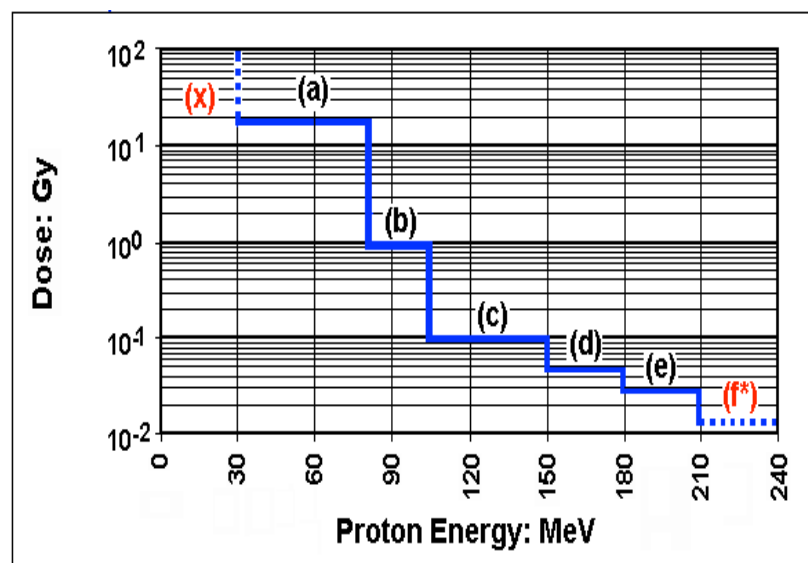

Figure 4. The 7-step histogram of the solar flare proton spectrum delivered to EBT 2 film from Proteus 230 medical cyclotron by manipulating the treatment planning system (TPS) commonly used in cancer therapy protocol. 
Due to the severe overload of the cyclotron ion source, we refrained from the delivery of highest dose (100 Gy) at lowest proton energy $(15 \mathrm{MeV})$, corresponding to $1^{\text {st }}$ histogram step (x).

After the completion of the proton exposure the EBT 2 film was scanned using an Epson XL 10000 scanner and subsequently evaluated with the well-known open source Image $\mathrm{J}$ software package and shown in Figure 5.

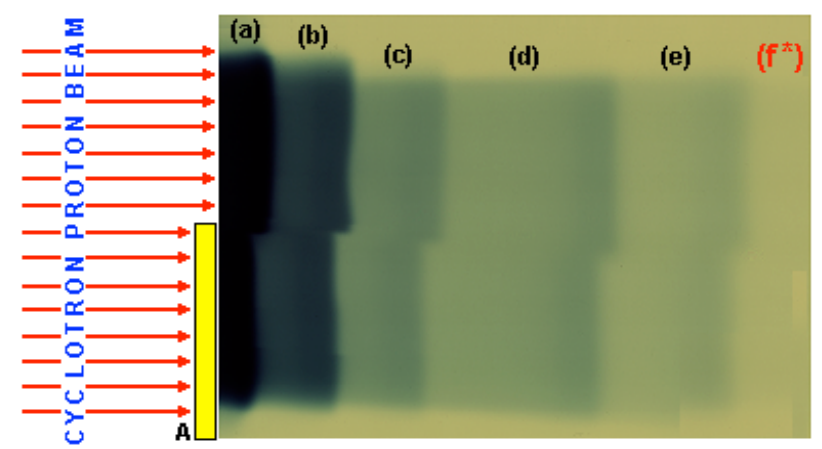

Figure 5. Showing the scanned photograph of the EBT 2 film. Five bands corresponding to proton energy histogram (Figure 4 ) are visible. Due to very low dose the $6^{\text {th }}$ band ( $\left.f^{*}\right)$ is not recognizable. The shift of the bands towards left (lower part) is due to proton energy loss by the $0.6 \mathrm{~cm}$ thick acryl plate (A).

The proton depth dose distribution along the EBT 2 film, corresponding to un-attenuated and attenuated with $0.6 \mathrm{~cm}$ acryl glass are depicted in Figure 6. The optical density of the background (penumbra) region was subtracted from the main zone of interest for proton dose calculations. A set of EBT 2 film samples was irradiated with protons with known doses in order to estimate the dose calibration constant used in this work [8]. Various investigators have validated the proton dose response linearity up to an exposure of $40 \mathrm{~Gy}$ [9].

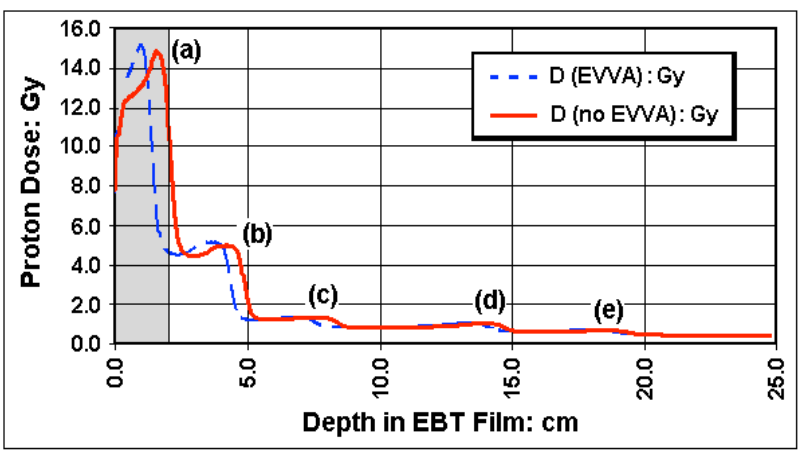

Figure 6. Simulated solar flare proton doses are plotted as functions of depth along the tissue equivalent EBT 2 film. The thick (solid-red) and thin (broken-blue) lines represent un-attenuated $\left(D_{\text {no EVVA }}\right)$ and with $0.6 \mathrm{~cm}$ thick acryl glass attenuated ( $\left.\mathrm{D}_{\mathrm{EVVA}}\right)$ conditions respectively. The energy bands (a), (b), (c), (d) and (e) are visible in Figure 5.

Evidently, the peaks along the above depth dose distribution curve indicate Bragg-Peak of individual energy bin (Figure 4), which are also visualized by the scanned EBT 2 film (Figure 5).

\section{Data analysis and results}

\subsection{Historical and simulated solar flare events}

The proton spectra of the historical solar flare events as shown in Figure 1 were analysed and using equations 1 and 2 we have calculated the corresponding proton dose rate in eye as well fluence average the LET (Figure 7).

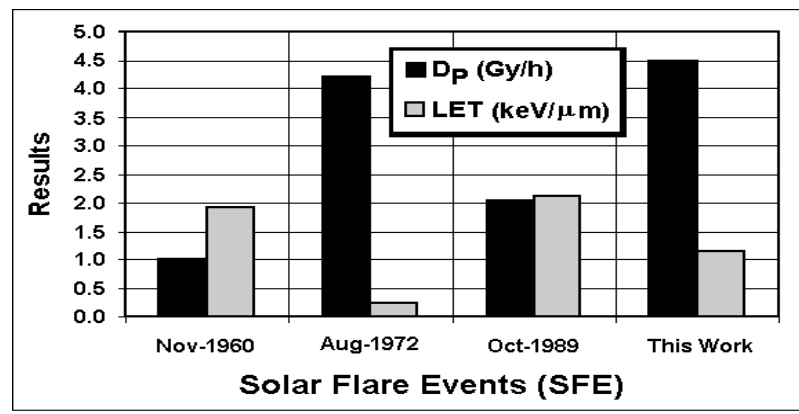

Figure 7. Showing the calculated un-attenuated proton dose rate $\left(D_{P}\right)$ and fluence average LET in the eye from historical solar flare events (Figure 1) as well as flare simulated at Proteus 230 medical cyclotron at WPE (This work).

\subsection{Proteus 230 cyclotron generated solar flare}

We have further analyzed the proton depth dose distribution in EBT 2 film irradiated at our cyclotron (Figures 3 ) and plotted the ratio of proton doses with and without implementation of the $6 \mathrm{~mm}$ thick acryl glass attenuator emulating the EVVA (indicated by "A" in Figure 5) as a function depth in EBT 2 film (Figure 8).

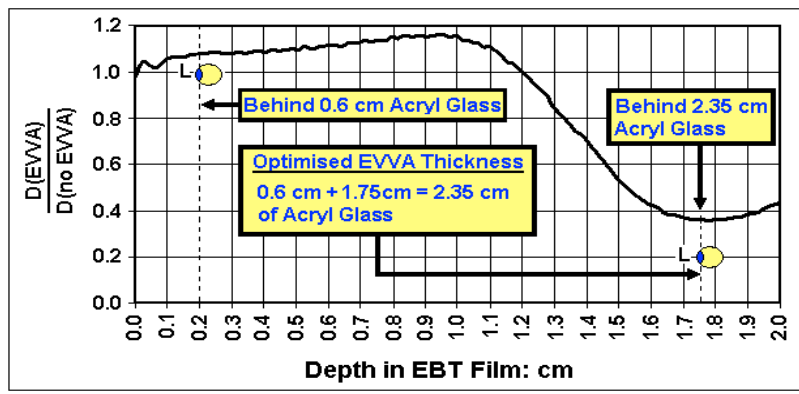

Figure 8. Showing the ratio $\left(\mathrm{D}_{\mathrm{EVVA}} / \mathrm{D}_{\mathrm{no}} \mathrm{EVVA}\right)$ at eye-lens (L) plotted as a function of depth in EBT 2 film.

The dotted vertical line on the left hand side indicates for a $6 \mathrm{~mm}$ thick acryl glass attenuator the value of proton dose ratio ( $\left.D_{\text {EVVA }}\right) / D_{\text {no EVVA }}$ ) found to be 1.07 at the depth of $0.2 \mathrm{~cm}$, i.e. the distance between lens from cornea (Figure 2). In fact, we see a dose build up instead of dose reduction. On the other hand, at a distance of $1.75 \mathrm{~cm}$ the dose ratio dropped to 0.36 . Therefore, by adding an extra $1.75 \mathrm{~cm}$ thick acryl glass plate, we obtained a total EVVA thickness of $2.35 \mathrm{~cm}$. Evidently, we shifted the point of minimum dose ratio to the location of the lens (L), allowing the predominant proton energy loss within the optimized EVVA, not in the lens. Here we have considered both acryl glass and radiochromic film EBT 2 are to be tissue equivalent [8]. 


\section{Summaries and conclusion}

Energetic particles ejected from sun's corona during solar flare events are primarily made of protons with an energy distribution spanning between several tens of $\mathrm{MeV}$ to around $1 \mathrm{GeV}$. However, the proton fluence relevant to high-energy $\left(E_{\mathrm{P}}>200 \mathrm{MeV}\right)$ tail of the solar flare spectra remains four orders of magnitude lower than the rest. This enables us to develop a solar flare spectra simulation set up using the therapeutic proton beam from a medical cyclotron delivering protons up to $230 \mathrm{MeV}$.

Solar flares occur sporadically, thereby imposing a high risk of radiation exposures to astronauts undertaking EVA outside the well-shielded space vehicle, i.e. the ISS. The human eye is one of the critical organs; highly vulnerable to radiation induced deterministic damage like cataract formation or even a complete blindness. Unlike astronaut's limbs and torso, which are well enclosed in adequately thick space suit, the eyes merely protected by a few millimeter thick Extravehicular Visor Assembly (EVVA) made of acryl glass. This motivated us to set up the above described simulated solar flare irradiation facility for efficacy testing of EVVA usually made of acryl glass. We have constructed a physical eye model using acryl glass plates and implemented the historical solar flare (spectra) for dose and LET calculations.

In a second study we have simulated a solar flare spectrum as a 7-step histogram using the $230 \mathrm{MeV}$ Medical-Cyclotron of WPE. The proton dosimetry was carried out using EBT 2 type radiochromic film. These EBT 2 films are tissue equivalent; hence, the depth dose distribution along the eye was directly estimated from the optical density of the film. The shielding efficacy (proton penetration) of a $0.6 \mathrm{~cm}$ thick acryl glass plate emulating the EVVA evaluated. The optimum thickness of the acryl glass EVVA was causing lowest proton dose in the lens calculated to be $2.35 \mathrm{~cm}$.

Evidently the optimal EVVA thickness depends on the spectral distribution of the SFE, which vary sporadically (Figure 1). Hence, a higher weight of the low-energy bin lowers the thickness of EVVA and vice versa. However, the implementation of a too thick EVVA is impractical in space flight, due to an increased payload. The radiation exposure to astronaut's eye could be substantially reduced abiding the ALARA (As Low As Reasonably Achievable), i.e. by reducing the time interval between SFE arrival and a swift retreat of the astronaut in the mother vehicle.

The secondary neutron and gamma ray production in proton irradiated soft tissue equivalent material is quite low, i.e., $0.002 \mathrm{~Sv} / \mathrm{Gy}$ (p) for $177 \mathrm{MeV}$ energy, as reported elsewhere [10]. Hence, in cases of solar flares, where much lower energy protons are predominant (Figure 1), the effects of secondary particles could be ignored. In the practice of proton therapy the beam-energy degraders and range shifters are the main sources unwanted background radiations. However, those parts are located close to the cyclotron, far away from the treatment rooms, hence, having no effects in the primary proton field (this work).

The fluence average proton LET $\sim 2.5 \mathrm{keV} / \mu \mathrm{m}$ (this work, Figure 7) found to be very close to that of ${ }^{60} \mathrm{Co}$ gamma rays, confirmed by TLD exposures [11, 12]. Hence, we have ignored LET dependence of EBT 2 film response (quench effect) in this work.

We have demonstrated that by manipulating the TPS [6], a common $230 \mathrm{MeV}$ Proton-Therapy cyclotron could be used to simulate solar flare proton spectra and utilized for testing of space suit components. The present set up is capable of irradiating a volume of $25 \times 25 \times 20 \mathrm{~cm}^{3}$, i.e. the size of an EVVA attached to astronaut's helmet with simulated solar flares. One can enhance this volume by moving the PPS along the $\mathrm{Y}$-axis in steps (Figure 3).

\section{Acknowledgements}

The authors wish to thank the IBA cyclotron operation team, in particular, Dr. rer. nat. Ann-Katrin Nix, Dipl. Ing (FH) Dennis Mantel and Dipl. Ing (FH) Tobias Mayer for their skillful technical supports.

\section{References}

[1] F. A. Cuchinotta, P. B. Saganti and M. R. Shavers (editors), Radiation Protection Studies of the International Space Station EVA Space Suits, NASA/TP-2003-212051 (2003).

[2] M. F. Moyers, P. B. Saganti and G. A. Nelson, EVA space suit proton and electron threshold energy measurements by XCT and range shifting, Radiat. Meas. 41(2006), pp. 1216-1226.

[3] E. W. Cascio and S. Sarkar, A solar flare simulation wheel for the radiation test beamline at the Francis H. Burr Proton Therapy Center, IEEE Trans. Nucl. Sci. 55(2008), pp. 3428-3434.

[4] B. M. Anderson, J. E. Nealy, M. H. Kim, G. D. Qualls and J. W. Wilson, Analysis of a radiation model of shuttle space suit, NASA/TP-2003-212158 (2003).

[5] M. A. Xapos, J. L. Barth, E. G. Stassinopoulos, S. R. Messenger, R. J. Walteres, G. P. Summers and E. A. Burke, Characterizing solar proton energy spectra for radiation effect applications, IEEE Trans. Nucl. Sci. 47(2000), pp. 2218-2223.

[6] IMPAC Medical System Inc., Elekta Radiation Treatment Planning System, XiO-LRNXIO04050, Release Note PDA 4.70.50 (2012).

[7] J. Valentin (editor), ICRP Publication 103, The 2007 Recommendations of the International Commission on Radiological Protection, Elsevier, (2008), pp. 82-98, ISBN 9780702030482.

[8] S. M. Vatnisky, Radiochromic film dosimetry for clinical proton beams, Appl. Radiat. Isot. 48(1997), pp. 643-651.

[9] A. Piermatti, R. Micheli, A. Fidanzio, S. De. Canne, C. D. Angelis, S. Onori, M. Pacillo, E. Petetti, L. Rafaele and M. G. Sabani, Radiochromic film 
Dosimetry of a low energy proton beam, Medical Physics 27(2000), pp. 1655-1660.

[10]U. Schneider, S. Agosteo, E. Pedroni and J. Besserer, Secondary neutron dose during proton therapy using spot scanning, Int. J. Rad. Oncol. Biol. Phys. 53 (2002), pp. 244-251.

[11] S.W.S. McKeever, Thermoluminescence of Solids,
The Cambridge University Press, (1988), pp. 213, ISBN 0521368111.

[12]B. Mukherjee, Light conversion efficiencies of ${ }^{7} \mathrm{LiF}$ Thermoluminescence dosimeters (TLD-700) for protons with a non-mono-energetic energy distribution, Nuclear Instruments and Methods 190(1981), pp. 207-208. 